\title{
COMMENTARY
}

\section{New Hope for Prevention of Preterm Delivery}

\author{
Drucilla J. Roberts
}

From the Department of Pathology, Massachusetts General Hospital, Boston, Massachusetts

\begin{abstract}
CME Accreditation Statement: This activity ("ASIP 2013 AJP CME Program in Pathogenesis") has been planned and implemented in accordance with the Essential Areas and policies of the Accreditation Council for Continuing Medical Education (ACCME) through the joint sponsorship of the American Society for Clinical Pathology (ASCP) and the American Society for Investigative Pathology (ASIP). ASCP is accredited by the ACCME to provide continuing medical education for physicians.
\end{abstract}

The ASCP designates this journal-based CME activity (“ASIP 2013 AJP CME Program in Pathogenesis") for a maximum of 48 AMA PRA Category 1 Credit(s) ${ }^{\mathrm{TM}}$. Physicians should only claim credit commensurate with the extent of their participation in the activity.

CME Disclosures: The authors of this article and the planning committee members and staff have no relevant financial relationships with commercial interests to disclose.

Preterm delivery (delivery before the 37 th week of gestation) accounts for more than $12 \%$ of all births in the United States and has increased $33 \%$ in the past 25 years. ${ }^{1}$ Preterm birth is a huge international problem, accounting for 1 million perinatal and pediatric deaths, and is the most significant contributor to perinatal and pediatric morbidity and mortality. ${ }^{2}$ The devastating outcomes that are increased in prevalence with premature delivery include blindness, chronic lung disease, cognitive neurodisability, cerebral palsy, and death. ${ }^{3}$ The prevalence of preterm delivery is increasing despite major advances in obstetric and neonatal care. ${ }^{4}$ There are many different etiologies of preterm delivery, indicated (or iatrogenic) and spontaneous, but the outcomes are similar. ${ }^{5}$ Available clinical interventions targeting prevention of spontaneous preterm delivery are few and have had limited success. ${ }^{6}$ One of the potentially clinically targetable causes of preterm delivery is inflammatory disorders, accounting for between $25 \%$ and $55 \%$ of obstetric precursors of preterm birth. ${ }^{4}$ Unfortunately, to date, no effective therapy to prevent inflammatory preterm birth is available. In this issue, Sundaram et $\mathrm{al}^{7}$ describe a novel use of a common organic solvent, N,N-dimethylacetamide (DMA), in preventing inflammation-mediated preterm birth.

The authors present results from both in vivo and in vitro studies showing the effects and describing the possible functions of DMA in preventing inflammation-mediated preterm delivery. To model placental inflammation in vivo the authors injected endotoxin [lipopolysaccharide (LPS) ${ }^{8}$ intraperitoneally into timed pregnant mice (embryonic day 15.5; gestational period, 19 to 21 days). This resulted in marked placental inflammation and preterm delivery within 15 hours. The authors administered a DMA injection after the
LPS injection and found that the preterm delivery rate and placental inflammatory grade significantly decreased in a dose-dependent manner. This was accompanied by reduced expression of specific proinflammation mediators known to be associated with preterm delivery in humans (matrix metalloproteins- 8 , IL-1B, tumor necrosis factor $\alpha$, and IL-6) ${ }^{9}$ and an increase in IL-10, which is thought to be immunoprotective of the fetal allograft. ${ }^{10}$ They then used in vitro systems to analyze the cellular function of DMA and found that in a macrophage cell line (RAQ264.7) stimulated with LPS, the DMA appeared to suppress macrophage bactericidal activity and activated the NF- $\kappa \mathrm{B}$ signaling pathway without negatively affecting cell viability. The authors achieved these results using a relatively inert, cheap, and available solvent. Could this be the holy grail of preventing premature delivery?

Although the results are encouraging and suggest a viable treatment for the prevention of preterm delivery, there are several caveats we must keep in mind. We describe three of them: the murine model, the inflammatory model, and the compound.

Human placentation is unique and difficult to model. The use of the mouse to model human obstetric pathologies is common because both are hemochorial and discoid, but differences are present and should not be ignored. One important difference is in the development and maturation of the placenta during gestation. Murine placental development is significantly slower and maturation is delayed when

Accepted for publication June 4, 2013.

Address correspondence to Drucilla J. Roberts, M.D., Department of Pathology, Massachusetts General Hospital, 55 Blossom St., Boston, MA- 02114., Phone: 16177241415, Fax: 617-7269312. E-mail: robertsd@ helix.mgh.harvard.edu 
compared with human placental development. ${ }^{11}$ The time point picked to study in this investigation, embryonic day 15.5 , which is approximately 30 weeks in humans using a temporal analogy only, is likely much less advanced in the mouse, physiologically and anatomically, and therefore may be more analogous to a much earlier gestational time point in humans. Whether this makes a difference in this study is unknown, the choice of the time point is not defended.

Another difference between mouse and human placentas is placental morphology. Human placenta is villous in structure whereas murine placenta is labyrinth, which is important to consider when studying compounds that may affect placental physiology or fetal exposure. ${ }^{11}$ The investigators show some evidence that DMA is nontoxic to the placenta itself (MTT assay ${ }^{12}$ ) or teratogenic to the fetus at the 24-hour time point examined (gross examination of the pups revealed no anomalies). Whether or not a longer exposure or prolonged gestation might result in placental or fetal pathology was not addressed. Although we were not provided with the metabolism or transplacental transport of DMA or its metabolites, other similar acetamides have shown fetal and placental effects, mainly growth restriction. ${ }^{13-15}$ Despite the lack of deleterious effect in their study, the authors accept the fact that high doses of DMA have been reported to show embryotoxicity in rats and rabbits. ${ }^{16,17}$ Longer time periods between exposure and delivery or examination therefore are needed.

Placental immunology is similar between the two systems and the usefulness of the murine system to study inflammatory and infectious disorders of pregnancy is well accepted. The effects of DMA was shown to have on inflammatory mediators likely are homologous to what we would expect in humans. Findings of decreased proinflammatory mediators and increased suppressors (IL-10) are exciting confirmatory data that DMA affects the immunoregulatory aspects of pregnancy maintenance, which is relevant for other less common pathologies, but perhaps are better targets for DMA treatment.

The LPS model of inflammation has some significant caveats when comparing it to inflammatory pathologies in humans. The results are based on a model of nonbacterial sepsis-like syndrome that the authors admit is not a good mimic of human pathology. The LPS model results in a systemic inflammatory response that includes inflammation of the placenta parenchyma, a model best suited for endotoxemia, not sepsis. ${ }^{18}$ Sepsis in pregnancy is a rare event, although it can be associated with preterm labor, which is a classic feature of septic abortion. ${ }^{19-21}$ The most important inflammatory disorder that results in spontaneous preterm delivery is acute chorioamnionitis, which often is associated with or complicated by preterm rupture of membranes. ${ }^{22}$ The diagnosis is made either clinically (fever, fetal tachycardia, uterine tenderness, foul smelling amniotic fluid) or pathologically (inflammation in the placental membranes with or without a fetal inflammatory response ${ }^{23}$ ). Acute chorioamnionitis, when caused by infection, is thought to be the sequelae of an ascending infection of the amniotic fluid by cervicovaginal flora (either resident or virulent) resulting in a local infection. It is characterized by a maternally derived inflammatory infiltrate in the placental membranes and chorionic plate, and often is accompanied by a fetal inflammatory response in the vessels of the placenta and umbilical cord. ${ }^{23,24}$ The LPS model of inflammation is a systemic one, not analogous to the local inflammation diagnostic of chorioamnionitis. It is perhaps more similar to acute villitis, a hematogenously based infection of the placenta resulting in neutrophilic involvement of the villi and intervillous space. Acute villitis is very rare and almost always infectious in etiology (personal experience), and is not a common cause of preterm delivery in humans.

The real problem that has eluded treatment in obstetric care of preterm delivery is acute chorioamnionitis. Chorioamnionitis is more akin to an abscess than sepsis or an organ/tissue infection (eg, pneumonia or cellulitis). The treatment for an abscess is drainage and not antibacterials or anti-inflammatories. In terms of pregnancy, treatment of chorioamnionitis is delivery. Continuing a pregnancy with infectious chorioamnionitis can lead to both fetal and maternal death. $^{21,25-29}$ Any treatment of infectious chorioamnionitis must be coupled with treating the infection. The effect of a DMA treatment muting the inflammatory response in a real infection such as chorioamnionitis has not been addressed in this study. Whether DMA treatment in typical infectious acute chorioamnionitis would reduce preterm delivery is unknown. There are many murine models of infectious placentitis that perhaps would be more relevant than the LPS model used in this study (see article by Krishnan et $\mathrm{al}^{30}$ and references therein). Use of DMA in these model systems more closely would mimic the human clinical situation.

The authors did not describe any fetal inflammatory response in the LPS model. The fetal inflammatory response is not an uncommon finding in association with the maternal infiltrate in acute chorioamnionitis and is thought to portend a worse prognosis for the fetus/neonate. ${ }^{31,32}$ This fetal response is not always a feature of congenital infection but a response to the maternal inflammatory response or one of stress. It is reasonable to ask if there was any fetal inflammation noted in this LPS model of placentitis.

Because no virulent organism is used in this model, it cannot be compared directly with an infectious chorioamnionitis and may be a better model for a noninfectious placentitis. There are inflammatory lesions of the placenta that are thought to be immunologically based (similar to allograft rejection reactions), for example, chronic villitis, ${ }^{33,34}$ chronic chorioamnionitis, ${ }^{35,36}$ and massive chronic intervillositis, ${ }^{37,38}$ all of which have a recurrence rate and are associated with perinatal morbidity, although not necessarily preterm delivery. Currently, there is no agreed on treatment for these sometimes catastrophic disorders. ${ }^{39}$ DMA possibly could provide some hope for treatment of these difficult pathologies.

This study did not follow up treated pregnancies through normal gestational periods. The authors did not investigate if there are any lasting effects after 24 hours, and did not allow any spontaneous deliveries of the pregnancies that 
survived the first 24 hours after treatment. Because inflammation is thought to play a critical role in labor onset ${ }^{40}$ this would be a very important point to study. It is possible that DMA might delay or inhibit normal parturition.

Despite such promising results, we need to temper enthusiasm over the potential application of DMA as a therapeutic option in treating inflammatory lesions of the placenta and preventing preterm delivery. There are concerns about the murine placenta as a model of human gestation, questions about the validity of the LPS system as a model of placentitis, and the lack of data about any effects of DMA on the placenta/fetus/maternal tissues past 24 hours of treatment. Yet, the promise of such an apparently safe and cheap compound that dampens or obviates maternal inflammatory pathology and inhibits preterm delivery is exciting enough. No other factor has shown such promise in terms of treating the inflammatory causes of preterm delivery.

\section{References}

1. Shapiro-Mendoza CK, Lackritz EM: Epidemiology of late and moderate preterm birth. Semin Fetal Neonatal Med 2012, 17:120-125

2. Blencowe HCS, Oestergaard M, Chou D, Moller AB, Narwal R, Adler A, Garcia CV: National, regional and worldwide estimates of preterm birth. Lancet 2012, 9:2162-2172

3. Iacovidou N, Varsami M, Syggellou A: Neonatal outcome of preterm delivery. Ann N Y Acad Sci 2010, 1205:130-134

4. Goldenberg RL, Culhane JF, Iams JD, Romero R: Epidemiology and causes of preterm birth. Lancet 2008, 371:75-84

5. Yang X, Zeng W: Clinical analysis of 828 cases of iatrogenic preterm births. J Obstet Gynaecol Res 2011, 37:1048-1053

6. Simhan HN, Caritis SN: Prevention of preterm delivery. N Engl J Med 2007, 357:477-487

7. Sundaram S, Ashby CRJ, Pedkson R, Sampat V, Sitapara R, Mantell L, Chen $\mathrm{C}-\mathrm{H}$, Yen $\mathrm{H}$, Abhichandani K, Munnangi S, Khadtare N, Stephani RA, Reznik SE: N, N-dimethylacetamide regulates the pro-inflammatory response associated with endotoxin and prevents preterm birth. Am J Pathol 2013, 183:422-430

8. Martich GD, Boujoukos AJ, Suffredini AF: Response of man to endotoxin. Immunobiology 1993, 187:403-416

9. Wei SQ, Fraser W, Luo ZC: Inflammatory cytokines and spontaneous preterm birth in asymptomatic women: a systematic review. Obstet Gynecol 2010, 116:393-401

10. Thaxton JE, Sharma S: Interleukin-10: a multi-faceted agent of pregnancy. Am J Reprod Immunol 2010, 63:482-491

11. Malassine A, Frendo JL, Evain-Brion D: A comparison of placental development and endocrine functions between the human and mouse model. Hum Reprod Update 2003, 9:531-539

12. Sargent JM: The use of the MTT assay to study drug resistance in fresh tumour samples. Recent Results Cancer Res 2003, 161:13-25

13. Macpherson ML, Giguere S, Hatzel JN, Pozor M, Benson S, Diaw M, Sanchez LC, Vickroy TW, Tell L, Wetzlich S, Sims J: Disposition of desfuroylceftiofur acetamide in serum, placental tissue, fetal fluids, and fetal tissues after administration of ceftiofur crystalline free acid (CCFA) to pony mares with placentitis. J Vet Pharmacol Ther 2013, 36:59-67

14. de Toranzo EG, Masana M, Castro JA: Administration of benznidazole, a chemotherapeutic agent against Chagas disease, to pregnant rats. Covalent binding of reactive metabolites to fetal and maternal proteins. Arch Int Pharmacodyn Ther 1984, 272:17-23

15. Gschwind HP, Schutz H, Wigger N, Bentley P: Absorption and disposition of 14C-labelled oxiracetam in rat, dog and man. Eur J Drug Metab Pharmacokinet 1992, 17:67-82
16. Okuda H, Takeuchi T, Senoh H, Arito H, Nagano K, Yamamoto S, Matsushima T: Developmental toxicity induced by inhalation exposure of pregnant rats to N,N-dimethylacetamide. J Occup Health 2006, 48: $154-160$

17. Stulla EF, Kraus WC: Embryotoxicity in rats and rabbits from cutaneous application of amide-type solvents and substituted ureas. Tox Appl Pharmacol 1977, 41:35-55

18. Rittirsch D, Hoesel LM, Ward PA: The disconnect between animal models of sepsis and human sepsis. J Leukoc Biol 2007, 81:137-143

19. Barton JR, Sibai BM: Severe sepsis and septic shock in pregnancy. Obstet Gynecol 2012, 120:689-706

20. Jewett JF: Septic induced abortion. N Engl J Med 1973, 289:748-749

21. Santamarina BA, Smith SA: Septic abortion and septic shock. Clin Obstet Gynecol 1970, 13:291-304

22. Martinelli P, Sarno L, Maruotti GM, Paludetto R: Chorioamnionitis and prematurity: a critical review. J Matern Fetal Neonatal Med 2012, 25(Suppl 4):29-31

23. Redline RW, Faye-Petersen O, Heller D, Qureshi F, Savell V, Vogler C: Amniotic infection syndrome: nosology and reproducibility of placental reaction patterns. Pediatr Dev Pathol 2003, 6: 435-448

24. Pankuch GA, Appelbaum PC, Lorenz RP, Botti JJ, Schachter J, Naeye RL: Placental microbiology and histology and the pathogenesis of chorioamnionitis. Obstet Gynecol 1984, 64:802-806

25. Nannini A, Weiss J, Goldstein R, Fogerty S: Pregnancy-associated mortality at the end of the twentieth century: Massachusetts, 19901999. J Am Med Womens Assoc 2002, 57:140-143

26. Lowenthal A, Katz M, Almog Y: Severe reversible myocardial depression in septic abortion. Acta Obstet Gynecol Scand 2006, 85: 502-503

27. Mabie WC, Barton JR, Sibai B: Septic shock in pregnancy. Obstet Gynecol 1997, 90:553-561

28. van Hoeven KH, Anyaegbunam A, Hochster H, Whitty JE, Distant J, Crawford C, Factor SM: Clinical significance of increasing histologic severity of acute inflammation in the fetal membranes and umbilical cord. Pediatr Pathol Lab Med 1996, 16:731-744

29. Monga M, Blanco JD: Intrauterine infection and preterm labor. Infect Dis Obstet Gynecol 1995, 3:37-44

30. Krishnan L, Nguyen T, McComb S: From mice to women: the conundrum of immunity to infection during pregnancy. J Reprod Immunol 2013, 97:62-73

31. Gomez R, Romero R, Ghezzi F, Yoon BH, Mazor M, Berry SM: The fetal inflammatory response syndrome. Am J Obstet Gynecol 1998 , 179:194-202

32. Romero R, Gomez R, Ghezzi F, Yoon BH, Mazor M, Edwin SS, Berry SM: A fetal systemic inflammatory response is followed by the spontaneous onset of preterm parturition. Am J Obstet Gynecol 1998, 179:186-193

33. Boog G: Chronic villitis of unknown etiology. Eur J Obstet Gynecol Reprod Biol 2008, 136:9-15

34. Redline RW: Villitis of unknown etiology: noninfectious chronic villitis in the placenta. Hum Pathol 2007, 38:1439-1446

35. Gersell DJ: Chronic villitis, chronic chorioamnionitis, and maternal floor infarction. Semin Diagn Pathol 1993, 10:251-266

36. Jacques SM, Qureshi F: Chronic chorioamnionitis: a clinicopathologic and immunohistochemical study. Hum Pathol 1998, 29:1457-1461

37. Jacques SM, Qureshi F: Chronic intervillositis of the placenta. Arch Pathol Lab Med 1993, 117:1032-1035

38. Boyd TK, Redline RW: Chronic histiocytic intervillositis: a placental lesion associated with recurrent reproductive loss. Hum Pathol 2000, 31:1389-1396

39. Doss BJ, Greene MF, Hill J, Heffner LJ, Bieber FR, Genest DR: Massive chronic intervillositis associated with recurrent abortions. Hum Pathol 1995, 26:1245-1251

40. Shynlova O, Lee YH, Srikhajon K, Lye SJ: Physiologic uterine inflammation and labor onset: integration of endocrine and mechanical signals. Reprod Sci 2013, 20:154-167 